\title{
Comprehensive Testing of Suspended Sediment Analysis Techniques to Support Monitoring Activities in the Danube River
}

\author{
Flóra POMÁZI ${ }^{\bowtie}$ and Sándor BARANYA \\ Budapest University of Technology and Economics, \\ Department of Hydraulic and Water Resources Engineering, Faculty of Civil Engineering, \\ Budapest, Hungary \\ $\bowtie$ pomazi.flora@emk.bme.hu
}

\begin{abstract}
Establishing and operating a harmonised sediment monitoring system along large rivers such as the Danube is a challenging international task. Our objective is to develop a time- and costeffective suspended sediment measurement protocol that can be easily carried out in the Upper-Hungarian Danube. In this study, we present the results of the comprehensive testing of direct and indirect (acoustic and optical) suspended sediment analysis methods, during which, we established moderate to strong relationships between the determined suspended sediment.
\end{abstract}

Keywords: acoustic backscatter, laser diffraction, suspended sediment, turbidimeter.

\section{INTRODUCTION}

Recent studies along the Danube (e.g. DanubeSediment 2019) pointed out the significance of harmonised suspended sediment (SS) monitoring system along large rivers. In the Upper-Hungarian Danube, the first Hungarian monitoring site with state-of-the-art instrumentation is currently under construction according to international guidelines and recommendations (e.g. Haimann et al. 2014). Our objective is to test different SS analysis methods in order to develop an integrated, time- and cost-effective SS measurement protocol, as the currently used monitoring method (pump sampling at 5 pre-fixed days per year; the suspended sediment concentration (SSC) determined by the evaporation method) is costly and time-consuming, and not suitable for detecting spatial and temporal variability of SS transport characteristics (i.e. SS load (SSL), SSC, particle size distribution (PSD)). As the monitoring site will consist of a near- 
bank turbidimeter and a horizontal-looking Acoustic Doppler Current Profiler (ADCP), we tested different acoustic and optical instruments and compared them with the filtration method.

\section{MEASUREMENT PROTOCOL}

During the field measurements, we took samples with an US P-61-A1 isokinetic sampler according to the multi-point method (in 5 verticals, in 5 measurement points per each). Additionally, an Acoustic Backscatter Sensor (ABS), the LISST-ABS was fixed on the isokinetic sampler to collect acoustic backscatter data from the same point as the physical sampling. In order to calculate the SSL, we carried out flow measurements with a $1200 \mathrm{kHz}$ ADCP instrument. This was supplemented with cross-sectional ADCP measurements. In order to determine the SSC, we tested different direct and indirect methods. We performed the followings: (i) positive pressure filtration $(0.45 \mu \mathrm{m})$, (ii) laser diffraction analysis using the LISST-Portable|XR, (iii) turbidity measurement using the VELP TB1 portable turbidimeter, (iv) in-situ acoustic backscatter data collection with the LISST-ABS, and (v) calibration of the ADCP relative backscatter.

\section{RESULTS AND DISCUSSION}

Strong relationships could be established between the filtration method and both the optical methods (LISST-Portable|XR: $R^{2}=0.94$, VELP: $R^{2}=0.97$ ), and the acoustical LISST-ABS $\left(R^{2}=0.89\right)$ which means that these indirect instruments can be calibrated with great reliability. However, we found that the reliability decreases above 150-200 mg/L (which occurs during high water regimes) in all three cases. The LISST-Portable|XR is an instrument that can provide information about the PSD as well. According to the results, at this section, the SS material is homogeneously distributed silt, with a mean size of 10-35 $\mu \mathrm{m}$. Based on our previous study on another Danube section (Pomázi and Baranya 2020), only the LISST-ABS measurements are expected to be sensitive to size effects within this range of particle size.

We examined how the LISST-ABS would work as a near-bank turbidimeter, and established a strong connection $\left(R^{2}=0.96\right)$ between the calibrated near-bank LISST-ABS concentration and the total cross-sectional SSL. It seems, that the future near-bank turbidimeter will be a suitable instrument of the SS monitoring indeed.

In case of the ADCP calibration, the established relationship is only moderate $\left(R^{2}=0.60\right)$ and more scattered which could be explained by the calibration process (Gartner 2004) itself as many parameters are needed to be determined (instrument-specific constants and coefficients that depend on water and sediment characteristics). However, we believe that the calibration can be used for qualitative analysis and thus, the spatial and temporal variability of SS monitoring can be enhanced.

\section{References}

DanubeSediment (2019), Sediment monitoring in the Danube River, Interreg Danube Project Report, available from: http://www.interreg-danube.eu/uploads/media/approved_project_output/0001/ 27/659489792a6c2b58c4e322ac8c609943565c3095.pdf.

Gartner, J.W. (2004), Estimating suspended solids concentrations from backscatter intensity measured by acoustic Doppler current profiler in San Francisco Bay, California, Mar. Geol. 211, 3-4, 169-187, DOI: 10.1016/j.margeo.2004.07.001. 
Haimann, M., M. Liedermann, P. Lalk, and H. Habersack (2014), An integrated suspended sediment transport monitoring and analysis concept, Int. J. Sed. Res. 29, 2, 135-148, DOI: 10.1016/ S1001-6279(14)60030-5.

Pomázi, F.; and S. Baranya (2020), Comparative assessment of fluvial suspended sediment concentration analysis methods, Water 12, 3, 873, DOI: 10.3390/w12030873.

Received 22 March 2021

Accepted 12 April 2021 